\title{
Involvement of mutant and wild-type CYSL TR2 in the development and progression of uveal nevi and melanoma
}

\author{
Rogier J. Nell ${ }^{1}$, Nino V. Menger ${ }^{1}$, Mieke Versluis ${ }^{1}$, Gregorius P. M. Luyten ${ }^{1}$, Robert M. Verdijk ${ }^{2,3}$, \\ Michele C. Madigan ${ }^{4,5}$, Martine J. Jager ${ }^{1}$ and Pieter A. van der Velden ${ }^{1 *}$
}

\begin{abstract}
Background: Activating $\mathrm{Ga}_{\mathrm{q}}$ signalling mutations are considered an early event in the development of uveal melanoma. Whereas most tumours harbour a mutation in GNAQ or GNA11, CYSLTR2 (encoding G-protein coupled receptor $C y s L T_{2} R$ ) forms a rare alternative. The role of wild-type $C y s L T_{2} R$ in uveal melanoma remains unknown.

Methods: We performed a digital PCR-based molecular analysis of benign choroidal nevi and primary uveal melanomas. Publicly available bulk and single cell sequencing data were mined to further study mutant and wildtype CYSLTR2 in primary and metastatic uveal melanoma.

Results: 1/16 nevi and 2/120 melanomas carried the CYSLTR2 mutation. The mutation was found in a subpopulation of the nevus, while being clonal in both melanomas. In the melanomas, secondary, subclonal CYSL TR2 alterations shifted the allelic balance towards the mutant. The resulting genetic heterogeneity was confirmed in distinct areas of both tumours. At the RNA level, further silencing of wild-type and preferential expression of mutant CYSLTR2 was identified, which was also observed in two CYSLTR2 mutant primary melanomas and one metastatic lesion from other cohorts. In CYSLTR2 wild-type melanomas, high expression of CYSLTR2 correlated to tumour inflammation, but expression originated from melanoma cells specifically.

Conclusions: Our findings suggest that CYSLTR2 is involved in both early and late development of uveal melanoma. Whereas the CYSLTR2 p.L129Q mutation is likely to be the initiating oncogenic event, various mechanisms further increase the mutant allele abundance during tumour progression. This makes mutant $C y s L T_{2} R$ an attractive therapeutic target in uveal melanoma.
\end{abstract}

Keywords: CYSLTR2, Uveal nevus, Uveal melanoma, Digital PCR, Heterogeneity

\section{Background}

Uveal melanoma is the most common primary tumour in the adult eye, with an annual incidence of 2-8 per million [1]. Up to $\sim 50 \%$ of the patients develops metastatic disease, predominantly to the liver. After hepatic spreading, uveal melanoma is usually fatal within a year [2].

\footnotetext{
* Correspondence: P.A.van der Velden@lumc.nl

'Department of Ophthalmology, Leiden University Medical Center, Leiden, the Netherlands

Full list of author information is available at the end of the article
}

The cancer originates from melanocytes in the uvea, the pigmented vascular layer of the eye. It is hypothesised that (epi)genetic alterations drive the transformation of these melanocytes into benign nevi and subsequently into malignant melanomas. However, it is rare to find histologically distinct pre-malignant lesions within or adjacent to a primary tumour [3].

Activating $\mathrm{G \alpha}_{\mathrm{q}}$ signalling mutations at mutuallyexclusive hotspots in GNAQ and GNA11 (p.Q209 and p.R183) are found in a large majority of uveal

(C) The Author(s). 2021 Open Access This article is licensed under a Creative Commons Attribution 4.0 International License, which permits use, sharing, adaptation, distribution and reproduction in any medium or format, as long as you give appropriate credit to the original author(s) and the source, provide a link to the Creative Commons licence, and indicate if changes were made. The images or other third party material in this article are included in the article's Creative Commons licence, unless indicated otherwise in a credit line to the material. If material is not included in the article's Creative Commons licence and your intended use is not permitted by statutory regulation or exceeds the permitted use, you will need to obtain permission directly from the copyright holder. To view a copy of this licence, visit http://creativecommons.org/licenses/by/4.0/ The Creative Commons Public Domain Dedication waiver (http://creativecommons.org/publicdomain/zero/1.0/) applies to the data made available in this article, unless otherwise stated in a credit line to the data. 
melanomas [4, 5]. Their clonal abundance in primary tumours suggests that these mutations are acquired early in tumour development [6]. The presence of these mutations in the majority of choroidal nevi supports this hypothesis [7].

The essential role of $\mathrm{G \alpha}_{\mathrm{q}}$ signalling in uveal melanoma tumorigenesis has been further supported by the discovery of rare but recurrent mutations in PLCB4 (p.D630) and CYSLTR2 (p.L129Q) $[8,9]$. These genes, encoding phospholipase $C \beta 4$ and a $\mathrm{G \alpha}_{\mathrm{q}}$-coupled receptor respectively, are typically mutated in GNAQ and GNA11 wildtype tumours, providing an alternative way of activating the $\mathrm{G \alpha}_{\mathrm{q}}$ signalling pathway $[10,11]$.

CYSLTR2 encodes for the G protein-coupled receptor cysteinyl-leukotriene receptor $2\left(\operatorname{CysLT}_{2} \mathrm{R}\right)$ which is, under wild-type conditions, involved in leukotrienemediated signalling [12]. Although $\mathrm{CysLT}_{2} \mathrm{R}$ has several well-studied functions (e.g. in alveolar inflammation) and can be expressed by a variety of cell types (e.g. eosinophils, basophils, neutrophils and macrophages), its natural role and origin in uveal melanomas remains unclear [13]. Mutant CYSLTR2 however leads to constitutive activation of endogenous $\mathrm{G \alpha}_{\mathrm{q}}$ signalling, thereby stimulating the same pathways as oncogenic mutations in GNAQ and GNA11 [10, 11].

In this study, we examined the presence of CYSLTR2 mutations in non-malignant choroidal nevi and primary uveal melanomas. By targeted analysis using digital PCR, an accurate quantification of both CYSLTR2 mutant and wild-type alleles could be achieved even in DNA samples of lower quality and quantity. In the CYSLTR2 mutant melanomas, we further assessed the genetic heterogeneity within tumours and investigated the allelic balance of CYSLTR2 at the RNA level. Expansion into publicly available bulk and single cell uveal melanoma sequencing data allowed to evaluate our findings in independent cohorts of primary and metastatic tumours and to study the role of wild-type CYSLTR2 in melanomas with a mutation in GNAQ, GNA11 or PLCB4 [13, 14].

\section{Methods}

\section{Sample collection, microscopy and DNA/RNA extraction}

As described earlier, 16 choroidal nevi were dissected from 13 formalin-fixed post-mortem human donor eyes, with consent from Lions NSW Eye Bank and approval from the University of Sydney and University of New South Wales Human Research Ethics Committee [7]. The donor eyes, with anterior segment (cornea, iris and lens) removed, were examined using a Leica M60 dissecting stereomicroscope (Leica Microsystems, Wetzlar, Germany), with direct white light illumination (no filter) and $0.8 \mathrm{x}$ objective. A ProgRes CT5 Digital Camera (Jenoptik, Jena, Germany) with ProgRes Capture Pro v2 8.8 software was used to image the donor eye cups. The images were assembled as 8-bit RGB images, with increased brightness and contrast only, using Photoshop CS software (Adobe Corporation, San Jose, USA). The tissues were paraffin-embedded and $7 \mu \mathrm{m} \mathrm{sec-}$ tions were cut and collected on SuperFrost Plus ${ }^{\text {Tu }}$ slides (Menzel-Gläser, Braunschweig, Germany), dried and stored prior to use for routine haematoxylin and eosin (H\&E) staining. H\&E stained sections were viewed in bright field illumination with no filters using an Olympus IX71 microscope (20x and 40x objectives, Olympus, Tokyo, Japan). Images were acquired with an Olympus DP73 digital camera and Olympus cellSens Standard Software v1.16. Image resolution was $2400 \times 1800$ pixels. The images were assembled as 8-bit RGB images, with increased brightness and contrast only, using Photoshop CS software (Adobe Corporation). DNA was extracted using the ReliaPrep FFPE (Formalin-Fixed Paraffin-Embedded) gDNA Miniprep System (Promega Corp, Madison, USA).

120 fresh-frozen tumour samples, obtained from primary uveal melanomas treated by enucleation between 1999 and 2011, were available from the Department of Ophthalmology, Leiden University Medical Center (LUMC). All patient samples were collected with informed consent and this study was approved by the LUMC Biobank Committee and Medisch Ethische Toetsingscommissie under no. B14.003/DH/sh and B20.026. DNA and RNA were isolated from $25 \times 25 \mu \mathrm{m}$ sections using the QIAamp DNA Mini Kit and RNeasy Mini Kit respectively (Qiagen, Hilden, Germany). cDNA was prepared from RNA using the iScript ${ }^{\mathrm{Tw}} \mathrm{cDNA}$ Sythesis Kit (Bio-Rad Laboratories, Hercules, USA). Total nucleic acid from microdissected tissue was isolated using the Siemens Tissue Preparation System (Siemens Healthcare, Erlangen, Germany) [15].

For all procedures, the manufacturer's instructions were followed.

\section{Digital PCR}

Digital PCR experiments were carried out using the QX200 $^{\text {tw }}$ Droplet Digital $^{\text {Ts }}$ PCR System (Bio-Rad Laboratories) following the protocols described earlier $[6,16,17]$. A methodical overview of all experimental setups is presented in Supplementary Data 1. Context sequences, PCR annealing temperatures and supplier information for all assays used are provided in Supplementary Table 1.

Raw digital PCR results were acquired using QuantaSoft (version 1.7.4, Bio-Rad Laboratories) and imported in the online digital PCR management and analysis application Roodcom WebAnalysis (version 1.9.4, available via https://webanalysis.roodcom.nl).

\section{Sequencing}

To validate the presence of the CYSLTR2 mutation, 20 ng DNA was analysed in $7 \mathrm{uL}$ experiment, using $3.5 \mathrm{uL}$ iQ SYBR Green Supermix (Bio-Rad Laboratories) and 
primers in a final concentration of $500 \mathrm{nM}$. The PCR was performed in a CFX384 Real-Time PCR system (Bio-Rad Laboratories), using the same protocol as for digital PCR. Raw PCR products were purified and analysed for 'Quick Shot Short' Sanger Sequencing at BaseClear, Leiden, the Netherlands. AB1-files were analysed using Roodcom SangerSeq Analysis (version 1.0, https:// sangerseq.roodcom.nl).

\section{Analysis of publicly available datasets}

Segmented and arm-level SNP array copy number data files from the 80 primary uveal melanomas included the TCGA cohort were downloaded from the Broad GDAC Firehose (https://gdac.broadinstitute.org) [13]. Aligned whole exome and RNA-sequencing files were obtained from the NCI Genomic Data Commons data portal (GDC; https://portal.gdc.cancer.gov). Heterozygous SNP's were identified using freebayes (v1.3.1) [18]. Allele-specific RNA expression was determined based on the balance between the CYSLTR2 mutation versus wild-type and the balance between variants from common SNP's in the transcribed CYSLTR2 gene. For both, the number of reads mapping to the specific variants were determined using samtools (v1.7) and freebayes (v1.3.1) $[18,19]$.

The clinical and molecular annotation of all samples was obtained from the original publication [13].

DNA and RNA levels of the CYSLTR2 mutation in metastatic uveal melanoma CheckMate-03820,035 were available from the recent study performed by Anagnostou et al. [20].

Processed single cell RNA sequencing data from 8 primary and 3 metastatic melanomas studied by Durante et al. (GSE139829) was downloaded from the Gene Expression Omnibus repository (www.ncbi.nlm.nih.gov/ geo/query/acc.cgi?acc $=$ GSE139829) [14]. The data set was analysed in $\mathrm{R}$ (v3.6.0) using the Seurat library (v3.1.4), following the methodology from the original study [21]. Uveal melanoma cells were identified based on gene expression of MLANA (Melan-A). Clustering was conducted using 10 principle components and a resolution parameter set to 0.5 , and was visualized in a two-dimensional tSNE plot.

\section{Code availability}

All custom scripts used in this study are available via https:/github.com.nl/rjnell/cysltr2.

\section{Results}

CYSLTR2 p.L129Q is subclonally present in a GNAQ/GNA11 wild-type nevus

Previously, 16 choroidal nevi from 13 post-mortem collected eyes were analysed for GNAQ and GNA11 hotspot mutations. In 15 cases such mutations were found, but always in a subfraction of the nevus cells [7]. In the same cohort we applied a custom digital PCR assay, which targets the CYSLTR2 p.L129Q wild-type and mutant allele specifically. In nevus $12 \mathrm{~B}$, the only one being GNAQ and GNA11 wild-type, we now identified this CYSLTR2 mutation, while only CYSLTR2 wild-type alleles were detected in other nevi (Fig. 1a and b).

The mutant allele fraction of CYSLTR2 p.L129Q in nevus $12 \mathrm{~B}$ was $18 \%$, representing a mutant cell fraction of $36 \%$. As an earlier Melan-A staining indicated a > 95\% nevus cell purity of the macrodissected tissue, it turns out that a subpopulation of the nevus cells was mutated (Fig. 1c) [7]. This low level of mutant alleles was corroborated by Sanger sequencing (Supplementary Figure 1).

\section{CYSLTR2 p.L129Q is found in 2/120 primary uveal melanomas}

A cohort of 120 archival primary uveal melanomas, treated by enucleation at the LUMC, was screened for the CYSLTR2 p.L129Q mutation. This mutation was found in 2 tumours $(\sim 2 \%)$, which were both GNAQ and GNA11 wild-type (Table 1). Remarkably, both cases showed a mutant allele fraction significantly higher than $50 \%$, being at odds with heterozygosity for the mutant allele (Fig. 2a). To resolve the exact genetic composition and evolutionary history of these two melanomas, multiple spatially separated samples of both tumours were investigated using duplex and multiplex digital PCR (Supplementary Data 1).

\section{Genetic composition of CYSLTR2 mutant melanoma 1 (PUM-1)}

PUM-1 was obtained from an 86-year-old woman with a large uveal melanoma localised to the ciliary body of the right eye. Clinical characteristics of this case are presented in Table 1. From this patient, one macrodissected (PUM-1A) and one microdissected (PUM-1B) area of the primary tumour were analysed.

Digital PCR for the CYSLTR2 p.L129Q mutation in PUM-1A revealed a mutant allele fraction of 55\% (Fig. 2a). Using our multiplex digital PCR setup (Supplementary Data 1), we identified loss of the wild-type CYSL TR2 allele (chromosome 13q) in $67 \%$ of the cells within the tumour mass (Fig. 2b). This was confirmed by the allelic imbalance of heterozygous common single nucleotide polymorphism (SNP) rs2057413 in SETDB2, located $\sim 776 \mathrm{~kb}$ telomeric from CYSLTR2 (Supplementary Data 1). Taking the chromosome 13q copy number alteration into account, the mutant cell fraction was calculated to be $74 \%$. Additional digital PCR experiments showed that losses of $1 \mathrm{p}$ and $16 \mathrm{q}$, and gain of $8 \mathrm{q}$ were also present in $\sim 74 \%$ of the cells. However, loss of chromosome $3 p$ was detected in $67 \%$ of the cells.

The comparable abundances ( $74 \%$ of total tumour) of the CYSLTR2 mutation, gain of $8 \mathrm{q}$ and losses of $1 \mathrm{p}$ 


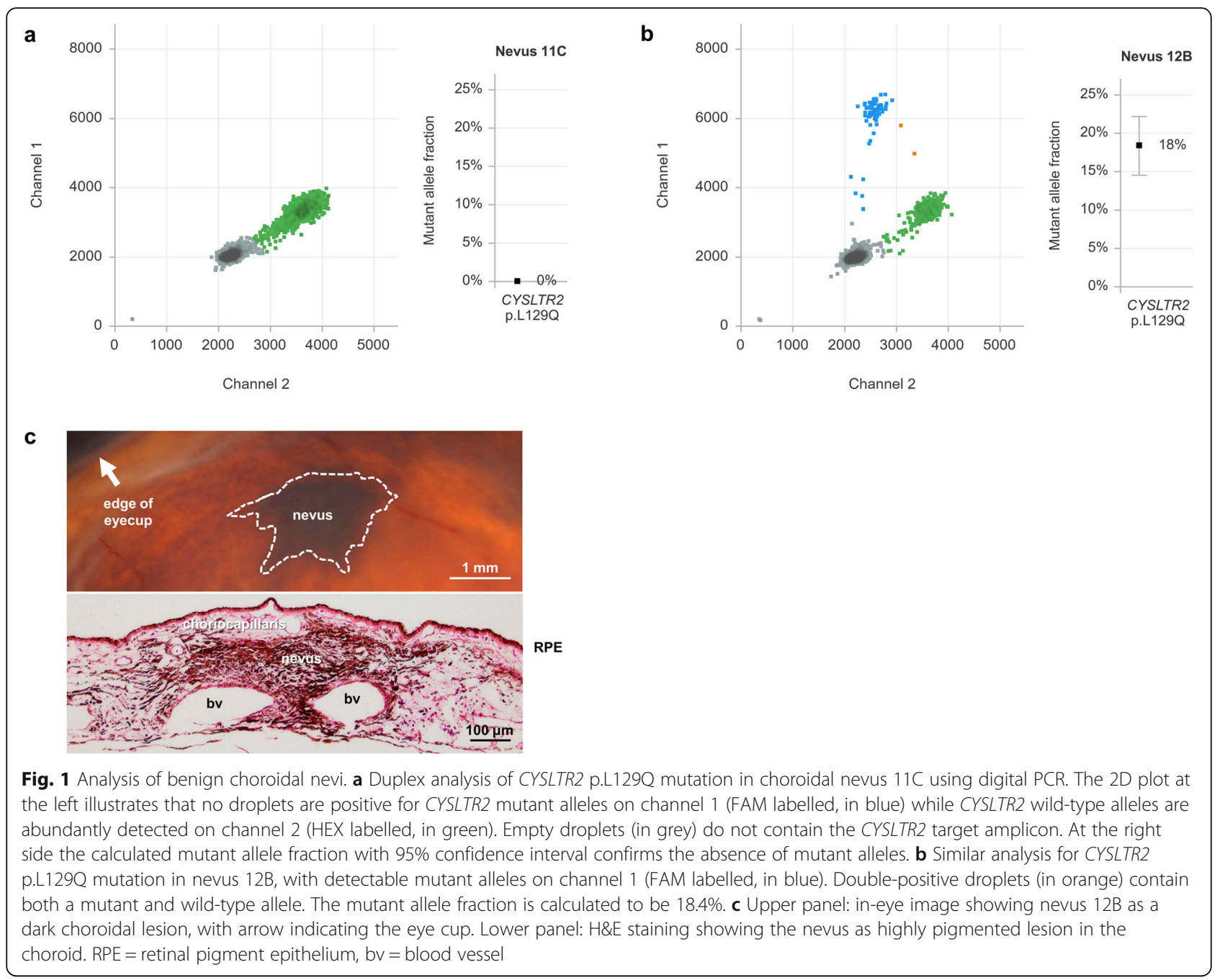

and $16 \mathrm{q}$ suggest that these alterations are clonally present in this sample and occurred during an early developmental phase. The losses of both wild-type CYSL TR2 and chromosome 3p were present in a significantly smaller, subclonal proportion of the cancer cells $(67 \%$ of total tumour), which indicates that these alterations occurred later during tumour development and that these cells coexisted with the predecessor clone. Taken together, the two clones represented 7 and $67 \%$ of the complete tumour mass, while another $26 \%$ of nonmalignant, healthy cells complemented the tumour composition (Fig. 2b-d and Supplementary Data 1).

Analysis of a melanoma cell-rich area of the primary tumour, obtained by microdissection (PUM-1B), was specified to the single-amplicon assays (CYSLTR2 mutation and SNP assays on chromosome $3 p$ and 13q) to prevent biases in DNA availablity due to degradation of FFPE-based input material. Again, two different clones were identified: in $99 \%$ of the cells the CYSLTR2 mutation was present, with chromosome $3 p$ and $13 q$ lost in $\sim 93 \%$ of the cells, giving clonal sizes of 6 and 93\%, and a healthy cell fraction of $1 \%$ (Fig. $2 \mathrm{~d}$ and Supplementary Data 1).

The genetic alterations of PUM-1 are typically associated with a poor prognosis, which is highlighted by the observed negative BAP1 nuclear staining (Table 1) [16]. This patient died of unknown cause 20 months after enucleation.

\section{Genetic composition of CYSLTR2 mutant melanoma 2 (PUM-2)}

PUM-2 presented as an iridociliary melanoma in an 8year-old male child, which led to enucleation of the right eye. An overview of the clinical characteristics is presented in Table 1. From this patient, one macrodissected (PUM-2A) and one microdissected (PUM-2B) area of the tumour were analysed.

In PUM-2A a CYSLTR2 p.L129Q mutant allele fraction of $57 \%$ was observed (Fig. 2a), which is also at odds with heterozygosity. In this tumour, a gain of 
Table 1 Clinical and molecular characteristics of CYSLTR2 mutant primary uveal melanomas (PUM-1, PUM-2, V4-A9ED, VD-AA80 and YZ-A982) analysed in this study

\begin{tabular}{|c|c|c|c|c|c|}
\hline \multirow[t]{2}{*}{ Case } & \multirow[t]{2}{*}{ PUM-1 } & \multirow[t]{2}{*}{ PUM-2 } & \multirow{2}{*}{$\begin{array}{l}\text { TCGA } \\
\text { V4-A9ED }\end{array}$} & \multirow{2}{*}{$\begin{array}{l}\text { TCGA } \\
\text { VD-AA80 }\end{array}$} & \multirow{2}{*}{$\begin{array}{l}\text { TCGA } \\
\text { YZ-A982 }\end{array}$} \\
\hline & & & & & \\
\hline Institute & $\begin{array}{l}\text { Leiden University } \\
\text { Medical Center }\end{array}$ & $\begin{array}{l}\text { Leiden } \\
\text { University } \\
\text { Medical } \\
\text { Center }\end{array}$ & $\begin{array}{l}\text { Institut Curie } \\
\text { France }\end{array}$ & $\begin{array}{l}\text { University of } \\
\text { Liverpool }\end{array}$ & Ohio State University \\
\hline Age at diagnosis & 86 & 8 & 45 & 77 & 79 \\
\hline Gender & female & male & male & male & female \\
\hline Tumour location & $\begin{array}{l}\text { choroid/ } \\
\text { ciliary body }\end{array}$ & $\begin{array}{l}\text { iris/ } \\
\text { ciliary body }\end{array}$ & $\begin{array}{l}\text { choroid/ } \\
\text { ciliary body }\end{array}$ & $\begin{array}{l}\text { choroid/ } \\
\text { ciliary body }\end{array}$ & $\begin{array}{l}\text { choroid/ } \\
\text { ciliary body }\end{array}$ \\
\hline $\begin{array}{l}\text { Tumour basal diameter in } \mathrm{mm} \\
\text { (clinical) }\end{array}$ & 18.63 & 8 & 17 & 17.3 & 23.64 \\
\hline Tumour thickness in $\mathrm{mm}$ (clinical) & 14.16 & 4 & 11 & 11.9 & 15.51 \\
\hline $\begin{array}{l}\text { Tumour basal diameter in } \mathrm{mm} \\
\text { (pathological) }\end{array}$ & 17 & $\begin{array}{l}\text { ring } \\
\text { melanoma }\end{array}$ & 16 & 13.5 & 25 \\
\hline $\begin{array}{l}\text { Tumour thickness in } \mathrm{mm} \\
\text { (pathological) }\end{array}$ & 11 & 4 & not available & not available & not available \\
\hline Extrascleral extension & $4.5 \mathrm{~mm}$ & absent & absent & absent & absent \\
\hline Tumour treatment & enucleation & enucleation & enucleation & enucleation & enucleation \\
\hline Vital status & dead & alive & alive & dead & dead \\
\hline $\begin{array}{l}\text { Time from diagnosis to follow-up in } \\
\text { days }\end{array}$ & 564 & 2850 & 1078 & 606 & 841 \\
\hline \multirow[t]{2}{*}{ Cause of death } & \multirow[t]{2}{*}{ not available } & \multirow[t]{2}{*}{ not applicable } & \multirow[t]{2}{*}{ not applicable } & metastatic & \multirow{2}{*}{$\begin{array}{l}\text { atrial fibrillation } \\
\text { complications }\end{array}$} \\
\hline & & & & uveal melanoma & \\
\hline Distant metastasis & not available & no & no & yes & no \\
\hline Locoregional Recurrence & not available & no & no & no & no \\
\hline AJCC primary tumour $(\mathrm{T})$ & pT3d & pT3 & T3b & T3a & T4b \\
\hline Cell type & mixed & mixed & spindle & epithelioid & spindle \\
\hline $\mathrm{Ga}_{\mathrm{q}}$ signalling mutation & CYSLTR2 p.L129Q & $\begin{array}{l}\text { CYSLTR2 } \\
\text { p.L129Q }\end{array}$ & CYSLTR2 p.L129Q & CYSLTR2 p.L129Q & CYSLTR2 p.L129Q \\
\hline BAP1 status & $\begin{array}{l}\text { aberrant } \\
\text { (loss of nuclear } \\
\text { staining) }\end{array}$ & $\begin{array}{l}\text { normal } \\
\text { (nuclear } \\
\text { staining) }\end{array}$ & $\begin{array}{l}\text { aberrant } \\
\text { (mutation) }\end{array}$ & $\begin{array}{l}\text { aberrant } \\
\text { (homozygous loss) }\end{array}$ & $\begin{array}{l}\text { normal } \\
\text { (no mutation) }\end{array}$ \\
\hline Chromosome $3 p$ status & loh & normal & loh & loh & gain \\
\hline Chromosome $8 \mathrm{q}$ status & gain & normal & normal & gain & gain \\
\hline
\end{tabular}

chromosome $13 q$ caused the relative excess of the mutant alleles (Fig. 2e and Supplementary Data 1). While the corrected mutant cell fraction was calculated to be $85 \%$, the gain of the mutant CYSLTR2 allele was quantified to be present in $68 \%$ of the cells, giving clonal sizes of 17 and $68 \%$, and a healthy cell fraction of $15 \%$ (Fig. $2 \mathrm{f}$ and Supplementary Data 1). No copy number alterations involving chromosome $1 p, 3 p, 8 q$ or $16 q$ were detected.

In PUM-2B, we also observed two clones: in $83 \%$ of all cells the CYSLTR2 p.L129Q mutation was identified, while $45 \%$ of the cells carried an extra copy of chromosome 13q. Accordingly, the two clones were present in 38 and $45 \%$ of the cells, and the remaining $17 \%$ represented healthy cells (Fig. 2f and Supplementary Data 1).

The copy number profile (normal chromosomes 3p and 8) of this tumour is correlated to a relatively good prognosis, which is supported by the positive $B A P 1 \mathrm{nu}-$ clear staining (Table 1) [16]. Indeed, after 8 years followup, no signs of metastatic spreading have been observed in this patient.

\section{Chromosome $13 q$ alterations are uncommon in uveal melanoma}

Since both CYSLTR2 mutant tumours presented with chromosome $13 \mathrm{q}$ aberrations, we questioned whether such copy number alterations are common in CYSL 


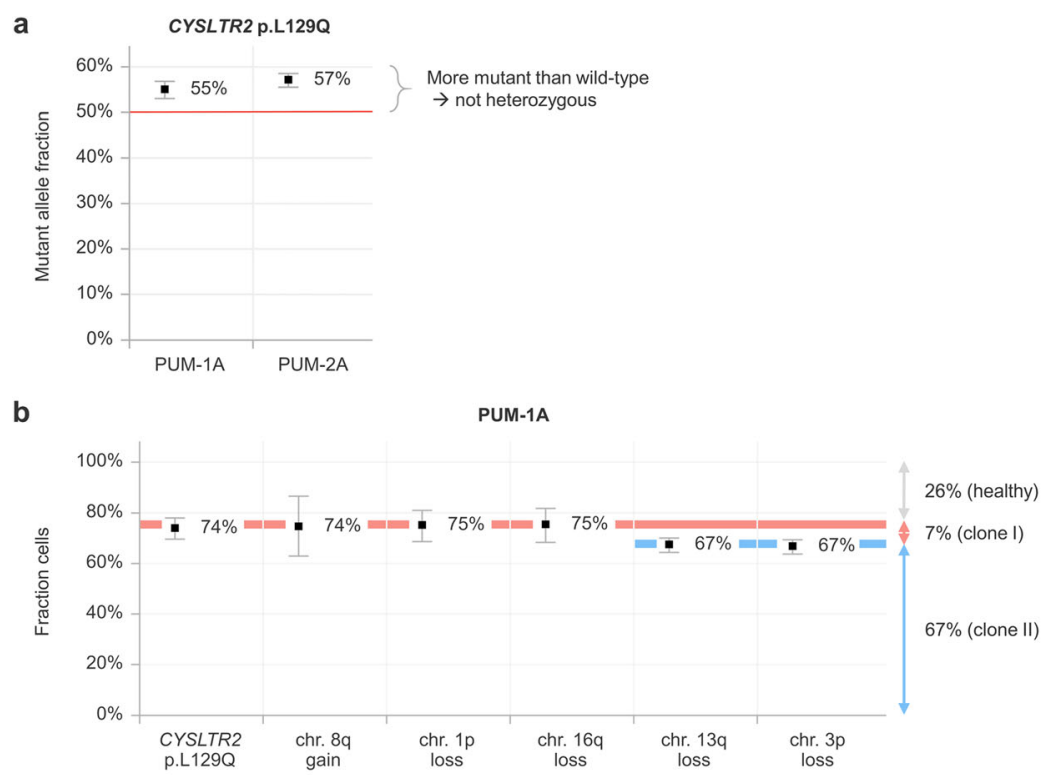

c

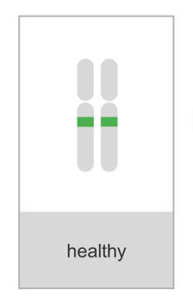

CYSLTR2 in PUM-1

e

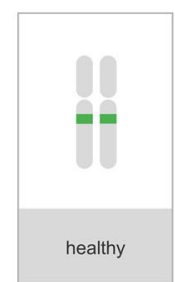

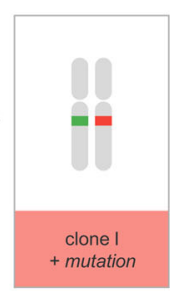

CYSLTR2 in PUM-2

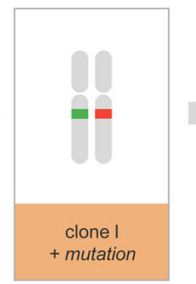

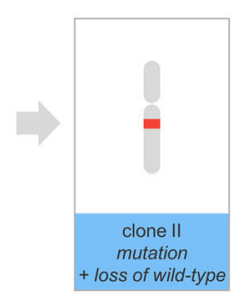

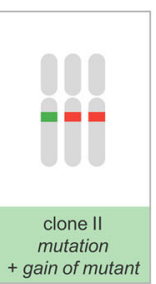

d

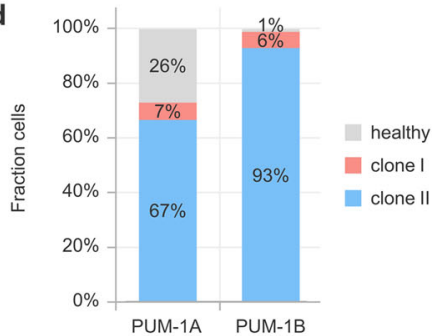

f

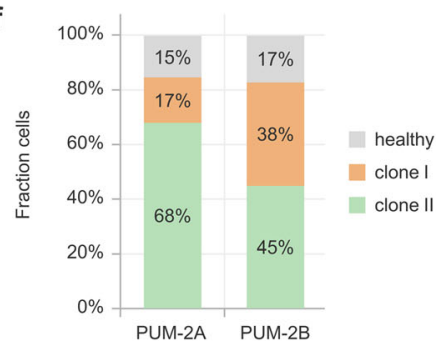

Fig. 2 Characterisation and molecular evolution of CYSLTR2 mutant uveal melanomas. a Mutant allele fractions for CYSLTR2 P.L129Q in PUM-1A and PUM-2A are both significantly higher than $50 \%$ and therefore in conflict with heterozygosity of this mutation. b Absolute quantification of genetic alterations in PUM-1A. CYSLTR2 mutation, gain of chromosome $8 q$ and losses of chromosome $1 p$ and 16q present being clonal in a fraction of $\sim 74 \%$ of total cells in the tumour mass. The losses of chromosome $13 q$ and chromosome $3 p$ are subclonal, as both are detected in $67 \%$ of the cells. c CYSLTR2 genetic evolution in PUM-1A/B, as inferred from absolute quantification of genetic aberrations. Starting at the germline situation (healthy cells), firstly the mutation has been acquired (clone I), after which the wild-type allele has been eliminated (clone II). $\mathbf{d}$ Clonal composition of PUM-1A/B shows the presence of both clones in both samples, but a lower fraction healthy cells is observed in the microdissected sample (PUM-1B: 1\%) versus the macrodissected sample (PUM-1A: 26\%). e CYSLTR2 genetic evolution in PUM-2A/B, as inferred from absolute quantification of genetic aberrations. Starting from the germline situation (healthy cells), firstly the mutation has been acquired (clone I), after which the mutant allele has been gained (clone II). $\mathbf{f}$ Clonal composition of PUM-2A/B shows the presence of both clones in both samples at varying levels

TR2 mutant and wild-type uveal melanomas. Therefore, we studied DNA sequencing and array genotyping data from 80 primary tumours from the TCGA (The Cancer Genome Atlas) cohort [13]. 3/80 cases presented with a CYSLTR2 p.L129Q mutation (V4A9ED, VD-AA8O and YZ-A982), of which the clinical details are summarised in Table 1 . In two of these tumours many chromosomal alterations were identified, suggesting a complex polyploid genotype (Supplementary Figure 2). However, a loss of the wild-type or gain of the mutant allele was not observed in these cases. 
In the 77 CYSLTR2 wild-type tumours, eight cases presented with a copy number alteration involving the CYSLTR2 locus at chromosome 13q, making it an uncommon event in uveal melanoma (Supplementary Figure 3).

\section{Mutant CYSLTR2 is preferentially expressed at the RNA level}

In the two CYSLTR2 mutant uveal melanomas from our Leiden cohort, we also evaluated the gene expression of both wild-type and mutant allele by digital PCR. Although an allelic imbalance could be expected based on the genetic alterations identified, hardly any wild-type allele expression was found, shifting the balance even more towards the mutant allele (Fig. 3a). Despite a lower level of significance, preferential expression of the mutant allele was also observed in two of the three CYSL TR2 mutant primary uveal melanomas from the TCGA cohort (Fig. 3b).

Very recently, Anagnostou et al. described the mutations and their expression in various types of metastatic melanomas studied in the CheckMate-038 (https://
clinicaltrials.gov/ct2/show/results/NCT01621490) clinical trial [20]. One uveal melanoma metastasis (case MUM20035) carried a copy number stable heterozygous CYSL TR2 mutation which was also hemizygously expressed at the RNA level (Fig. 3c).

To gain insight into the regular transcriptional allelic balance of CYSLTR2, we studied RNA sequencing data from 77 CYSLTR2 wild-type primary uveal melanomas from the TCGA cohort. The heterozygous presence of common SNPs illustrated a balanced biallelic expression under wild-type conditions (Fig. 3d). In tumour V4A9E8 a chromosome 13q copy number gain led to an allelic imbalance involving the CYSLTR2 locus, which was proportionately expressed at the RNA level (Fig. 3e).

It was however notable that the allelic balance of CYSL TR2 could not be studied in all samples, as CYSLTR2 expression varied largely between the melanomas [13].

\section{Wild-type CYSLTR2 is expressed by melanoma cells in} inflamed tumours

To investigate this variable CYSLTR2 expression, we further analysed the 77 primary melanomas from the

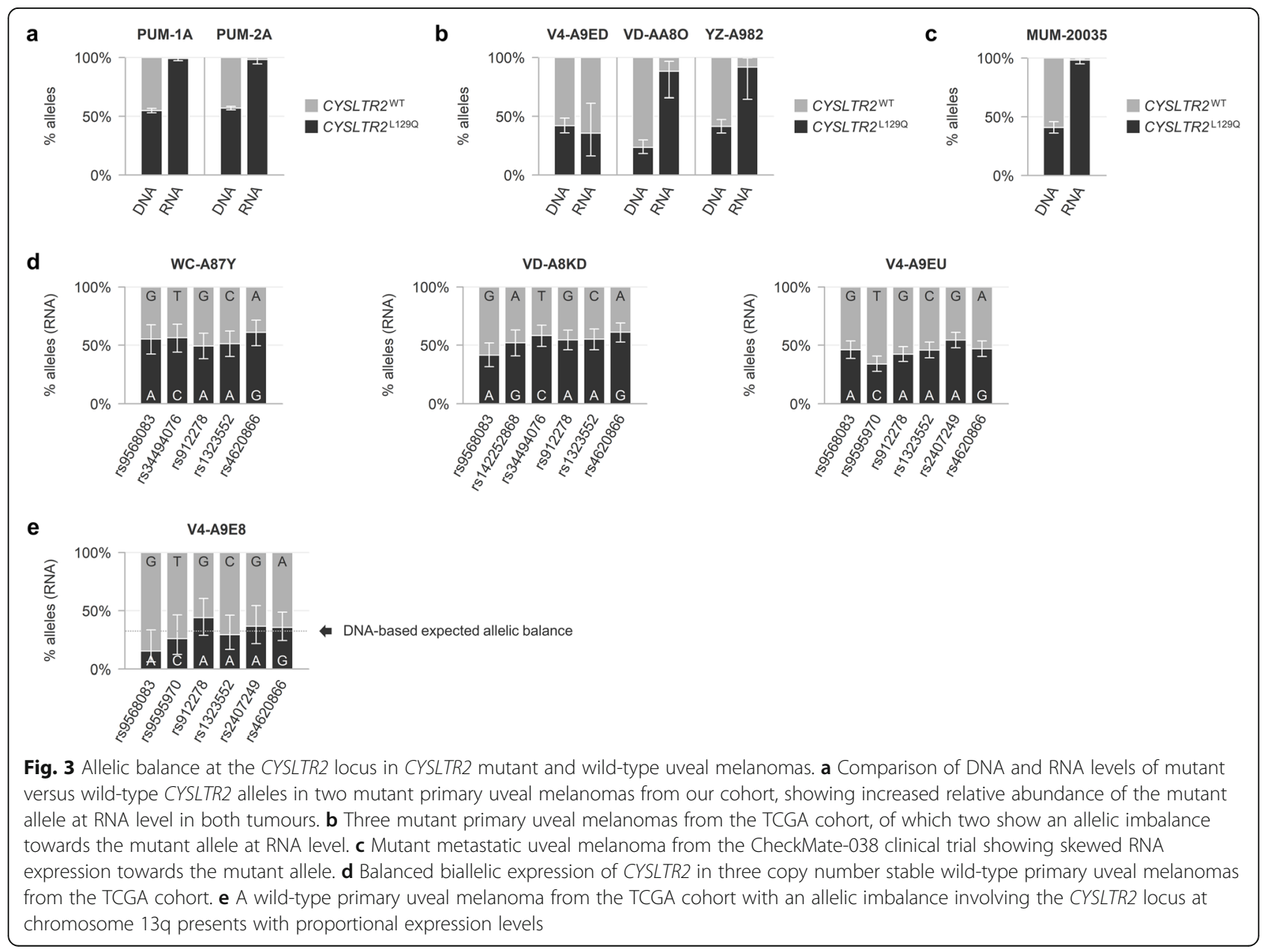


TCGA cohort and extended the analysis with the eight primary and three metastatic melanomas studied using single cell RNA sequencing as described by Durante et al. [13, 14]. All these melanomas were wild-type for CYSLTR2 and the majority carried mutations in GNAQ, GNA11 or PLCB4.

Based on bulk RNA data from the TCGA cohort, the highest levels of wild-type CYSLTR2 expression were observed in tumours with an inflammatory phenotype as shown by their immune gene expression signature (high expression of $C D 14, C D 163$ [monocytes/macrophages] and CD3D, CD8A [T cells]) (Fig. 4a). As CYSLTR2 is known to be expressed by a variety of immune cells [12], we used the single cell RNA sequencing data from the Durante cohort to delineate the cellular origin of this wild-type CYSLTR2 expression. In 6/11 cases CYSLTR2 was expressed in at least $2 \%$ of the cells, with percentages of up to more than $30 \%$ (Fig. 4b). Overall, the large majority of CYSLTR2 expression originated from melanoma cells.

As demonstrated in UMM066, CYSLTR2 expression was found in melanoma cells that also presented with high expression of pigmentation genes (MITF and TYR) and marker genes of MAPK (Mitogen-Activated Protein Kinase) pathway activation (MPAS) (Fig. 4c and Supplementary Figure 4) [22]. As leukotrienes, the ligands of $\mathrm{CysLT}_{2} \mathrm{R}$, cannot be measured at a single cell RNA-level, we used ALOX5AP and ALOX5 as pseudomarkers [12]. These genes, encoding enzymes catalysing the synthesis of leukotrienes, were specifically expressed by tumourassociated immune cells. Similar observations were made in the other tumours, suggesting that the immune infiltrate is equipped to produce the ligand for $\operatorname{CysLT}_{2} \mathrm{R}$ (Supplementary Figure 4).

\section{Discussion}

The majority of uveal melanomas are characterised by an activating $\mathrm{G \alpha}_{\mathrm{q}}$ signalling mutation [4, 5]. Whereas most tumours harbour a hotspot mutation in GNAQ or GNA11, the CYSLTR2 p.L129Q mutation forms a rare alternative [9]. In this study, we used digital PCR to analyse this mutation in non-malignant choroidal nevi and primary uveal melanomas. The potential role of wildtype CYSLTR2 in GNAQ, GNA11 or PLCB4 mutant uveal melanomas was studied in publicly available bulk and single cell sequencing data.

Previously, mutually-exclusive mutations in GNAQ and GNA11 (p.Q209L and p.Q209P) were found in 15/16 choroidal nevi [7]. In the one remaining non-mutated nevus (no. 12B), we now identified CYSLTR2 to be mutated (p.L129Q) (Fig. 1). In the earlier study, another nevus from the same eye (no. 12A) showed a GNA11 p.Q209L mutation [7]. Taken together, these findings indicate that mutations in GNAQ, GNA11 and CYSLTR2 - all recurrently found in uveal melanomas - can also present as an independent somatic event in benign uveal nevi.

In cutaneous epidermal melanocytes, the BRAF p.V600E mutation - an established oncogenic driver of cutaneous melanoma - is considered sufficient to initiate nevus formation. This is supported by the frequent presence of this mutation in nevus cells [7, 23-25]. The analysis of matched malignant and pre-malignant cutaneous lesions confirmed that a benign nevus may be a precursor of cutaneous melanoma [26]. Likewise, it may now be hypothesised that $\mathrm{G} \alpha_{\mathrm{q}}$ signalling mutations in GNAQ, GNA11 and CYSLTR2 represent a first step in uveal melanomagenesis.

We detected the CYSLTR2 mutation in 2/120 ( 2\%) screened primary uveal melanomas. This low prevalence reaffirms the rarity of this variant [9]. Both tumours in our study did not have a mutation in any of the hotspots of GNAQ or GNA11, confirming the mutual-exclusivity of these mutations.

Further analysis showed that the CYSLTR2 p.L129Q was clonal in both of these uveal melanomas, supporting an acquisition of this mutation early during tumour development. However, in both melanomas secondary copy number alterations of the CYSLTR2 locus (chromosome 13q) were observed as well: PUM-1 showed loss of the wild-type allele, while in PUM-2 the mutant allele was gained. These alterations were present in subclones of the melanomas, indicating that they arose later in the tumour evolution (Fig. 2).

At the RNA level, the CYSLTR2 allelic imbalance was skewed even further towards the mutant: in both melanomas hardly any expression of the wild-type allele was observed (Fig. 3a). Although in one case (PUM-1) this may be attributed to the elimination of the wild-type allele during tumour progression, in the other case (PUM2), a wild-type copy of CYSLTR2 was still available. Similarly, in two of the three CYSLTR2 mutant primary uveal melanomas from the TCGA cohort preferential expression of the mutant allele was observed, while in all cases the wild-type allele was still present (Fig. 3b). This suggests that besides genetic mechanisms epigenetic mechanisms might also lead to an increased relative abundance of mutant CYSLTR2.

In CYSLTR2 wild-type primary melanomas from the TCGA cohort, alterations involving chromosome 13q were uncommon and allelic balance was maintained in informative cases (Fig. 3d-e). This supports that the CYSLTR2 allelic imbalances at DNA and RNA levels are indeed associated with the p.L129Q mutation specifically. Whether these secondary alterations hold an additional metastatic risk, is - given the rarity of this mutation - still to be determined. However, the observation has been made that the mutation and secondary alterations involving CYSL 
a

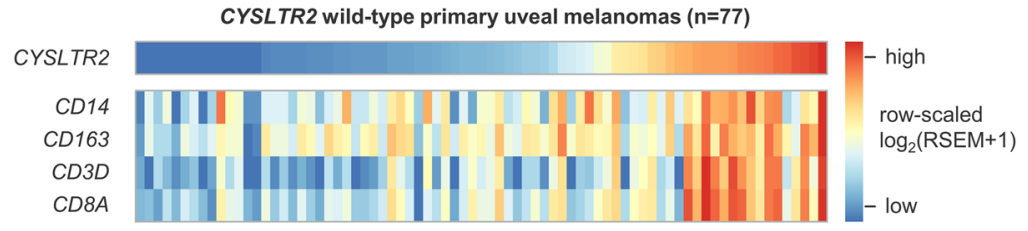

b

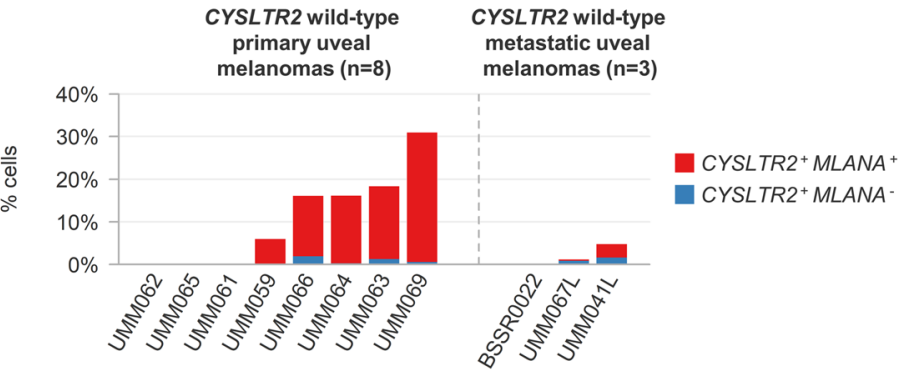

C
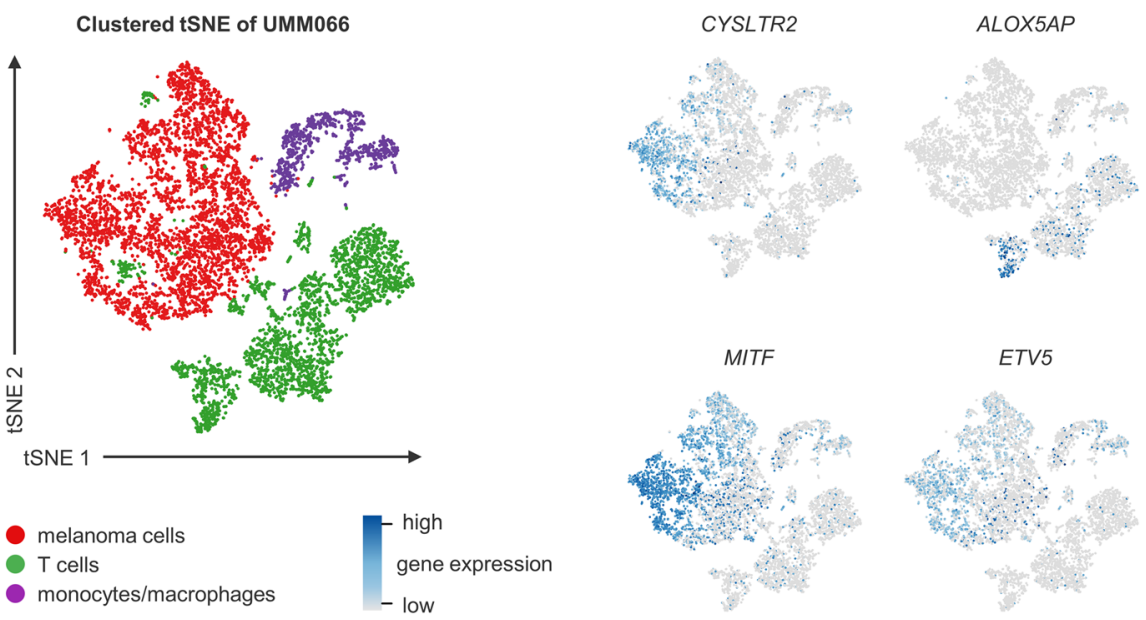

Fig. 4 Origin of wild-type CYSLTR2 expression in uveal melanomas analysed by bulk and single cell RNA sequencing. a Heatmap of row-scaled, normalised $\log _{2}(R S E M+1)$ gene expression levels of CYSLTR2, CD14 and CD163 (monocytes/macrophages) and CD3D and CD8A (T cells) as determined by bulk RNA sequencing in 77 wild-type primary uveal melanomas from the TCGA cohort. The highest CYSLTR2 expression levels are observed in the tumours with this immune gene expression signature. b Percentage CYSLTR2 expressing cells per tumour annotated for cell type, as determined by single cell RNA sequencing in eight primary and three metastatic uveal melanomas from the Durante cohort. In 6/11 tumours CYSLTR2 was expressed in $>2 \%$ of the cells, which were predominantly melanoma cells. c tSNE plot of single cell RNA sequencing data derived from UMM066, with annotation of clusters for cell type and expression levels per cell of CYSLTR2 (leukotriene receptor), MITF (pigmentation), ETV5 (MAPK activation) and ALOX5AP (leukotriene synthesis). CYSLTR2 was expressed in a subpopulation of melanoma cells that presented with high expression of genes involved in pigmentation and marker genes of MAPK activation. Enzymes catalysing the synthesis of leukotrienes (ligand of Cys $L T_{2} R$ ) are expressed by tumour-associated immune cells. Complete tSNE gene expression plots are presented in Supplementary Fig. 4

TR2 are found in all different molecular subtypes of uveal melanoma (Table 1).

Recently, the CYSLTR2 mutation was also detected in metastasising uveal melanomas [20, 27-29]. None of those cases displayed chromosome 13q copy number alterations involving CYSLTR2, but for one metastatic lesion we were able to analyse the CYSLTR2 allelic balance in detail (Fig. $3 \mathrm{c})$. While the mutation was heterozygous at DNA level, the RNA expression was skewed towards the mutant allele, in line with our observations in the primary tumours.
Again, our findings regarding CYSLTR2 p.L129Q show similarities to BRAF p.V600E in cutaneous melanoma. Besides a primary role in driving the development of nevi and melanomas, secondary genetic or transcriptomic alterations are also recurrently present. Along with the progression from nevus to (metastatic) melanoma, absolute and relative copy-number increases and proportionally higher expression levels of the mutant allele are observed, enhancing oncogenic signalling [30]. This possibly explains why therapies targeting mutant 
$B R A F$ (a driver mutation already present in the premalignant cutaneous nevus) have been so successful in the treatment of metastatic melanoma [31]. In analogy, mutant $\mathrm{CysLT}_{2} \mathrm{R}$ could be an attractive drug target for metastatic uveal melanoma. However, whereas effective antagonists for the other cysteinyl-leukotriene receptor $\left(\right.$ CysLT $\left._{1} \mathrm{R}\right)$ are already in clinical use (i.e. montelukast), a novel compound functioning as an inverse agonist is needed to inhibit the constitutively activated mutant CysLT $_{2} \mathrm{R}[9,10,32]$.

In bulk RNA from CYSLTR2 wild-type uveal melanomas, we observed variable expression of CYSLTR2, with the highest levels in tumours with an immune gene expression signature (Fig. 4a). These melanomas are characterised by a high infiltration of immune cells and are therefore seen as having an inflammatory phenotype [33, 34]. Given that a variety of immune cells is known to express CYSLTR2 [12], we investigated the cellular origin of this expression in these tumours. Using single cell RNA data, we confirmed that wild-type CYSLTR2 is mainly expressed by melanoma cells (Fig. 4b-c). As subpopulations of tumour-associated immune cells expressed ALOX5AP and ALOX5 (encoding the enzymes that catalyse the leukotriene synthesis), the ligand for wild-type $\mathrm{CysLT}_{2} \mathrm{R}$ may be produced by immune cells in the inflammatory microenvironment (Fig. 4c and Supplementary Figure 4). We hypothesise that via this ligand, tumour-associated immune cells may interact with the uveal melanoma cells and further promote tumour growth.

Constitutive activation of the receptor (as seen with the p.L129Q mutation) leads to oncogenic signalling, cell proliferation and enforcement of a melanocyte-lineagespecific transcriptional program in vitro and in vivo $[9$, 10]. Similarly, associations with a heavily pigmented phenotype have been reported in other melanocytic neoplasms carrying the p.L129Q mutation [35]. It is interesting to speculate which effects leukotriene-driven activation of wild-type CysLT ${ }_{2} R$ would have, as - based on our analysis of single cell data - wild-type CYSLTR2 expressing cells presented with high expression of genes involved in pigmentation and MAPK pathway activation (Fig. 4c and Supplementary Figure 4). Recently, it was shown in vitro that upon leukotriene activation wildtype $\mathrm{CysLT}_{2} \mathrm{R}$ can also signal through $\mathrm{G \alpha}_{\mathrm{q}}$ and activate the MAPK pathway [10]. Further research should evaluate whether such activation indeed has an extra oncogenic effect in these uveal melanoma cells which do not have a CYSLTR2 mutation, but carry an activating $G \alpha_{\mathrm{q}}$ signalling mutation downstream in GNAQ, GNA11 or PLCB4. As a consequence, wild-type $\mathrm{CysLT}_{2} \mathrm{R}$ might also represent a therapeutic target in uveal melanoma.

In contrast, mutant $\mathrm{CysLT}_{2} \mathrm{R}$ appeared unresponsive to leukotriene stimulation [10] and we observed that expression of the wild-type allele was downregulated in mutant tumours. This argues against a similar effect of the microenvironment in CYSLTR2 mutant melanomas.

Very recently, Slater et al. confirmed that wild-type $\mathrm{CysLT}_{2} \mathrm{R}$ is expressed in primary uveal melanomas and in primary and metastatic uveal melanoma cell lines [36]. While $\operatorname{CysLT}_{2} \mathrm{R}$ antagonist HAMI 3379 showed negligible effect on the viability or proliferation of the cell lines, it would be interesting to further study this drug in combination with leukotriene-driven activation of wild-type $\mathrm{CysLT}_{2} \mathrm{R}$.

Importantly, the oncogenic alterations in uveal melanoma are not restricted to the CYSLTR2 mutation. Therefore, we further studied the genetic heterogeneity of the two mutant melanomas from our cohort by digital PCR on multiple macro- and microdissected samples. In PUM-1 we found that the loss of wildtype CYSLTR2 - together with the loss of chromosome $3 p$ - was subclonally present and occurred after the CYSLTR2 mutation, losses of chromosome $1 \mathrm{p}$ and $16 \mathrm{q}$ and gain of chromosome 8q. PUM-2 presented as a relatively stable tumour, but with a chromosome 13q alteration (leading to gain of mutant CYSLTR2) which also existed in a tumour subclone. In both melanomas the presence of the distinct clones was confirmed in spatially separated areas of the tumour, indicating that the clones coexist throughout different parts of the tumour (Fig. $2 \mathrm{~d}$ and $\mathrm{f}$ ).

Hereby, we showed how the genetic heterogeneity and clonal evolution of a tumour can be construed from a deep quantitative analysis of bulk DNA, even in samples of lower DNA quality and quantity (i.e. FFPE-derived microdissections). The digital PCR experiments allowed us to analyse thousands of alleles per target, providing the statistical power to identify small allelic imbalances. Consequently, we identified differences in cancer cell populations which would not have been detectable using bulk sequencing-based techniques at conventional depths.

Whereas the genetic alterations in primary and - most recently - metastatic uveal melanoma have been largely catalogued, their relative order of occurrence in early tumoral development remains incompletely understood [27, 28, 37]. Moreover, the existence of intra-tumoral heterogeneity in primary uveal melanoma is still a topic of debate [6, 14, 27, 28, 37, 38]. Our study exemplifies that these important questions may also be answered on bulk tissue samples by accurate quantitative analysis using digital PCR, presenting a useful addition to the single cell-based approaches (mass cytometry, single-cell sequencing) that are currently dominating the field.

\section{Conclusions}

In conclusion, our findings strongly nominate the rare CYSLTR2 p.L129Q mutation as an early oncogenic event 
in GNAQ and GNA11 wild-type uveal nevi and melanomas. In mutant melanomas, the relative gene dosage of the CYSLTR2 mutant allele may be further increased during (metastatic) progression of the tumour. This makes these tumours promising candidates for mutant CysLT ${ }_{2} \mathrm{R}$-targeted therapy.

Moreover, we identified elevated expression of wildtype CYSLTR2 in inflamed tumours carrying a mutation in GNAQ, GNA11 or PLCB4. As this expression originated from the melanoma cells specifically, wild-type CysLT $_{2} \mathrm{R}$ could also be considered as a therapeutic target.

\section{Supplementary Information}

The online version contains supplementary material available at https://doi. org/10.1186/s12885-021-07865-x.

Additional file 1: Supplementary Table 1. Context sequences, PCR annealing temperatures and supplier information for all assays used. Supplementary Data 1. Methodical overview of all digital PCR experimental setups. Supplementary Figure 1. CYSLTR2 p.L129Q mutation detected by Sanger sequencing of choroidal nevus 12B compared to the reference sequence from a healthy control.

Supplementary Figure 2. SNP-array copy number profile and B-allele fractions of the CYSLTR2 mutant melanomas from the TCGA cohort. Supplementary Figure 3. Distribution of arm-level copy number alterations in 77 CYSLTR2 wild-type uveal melanomas from the TCGA cohort. Supplementary Figure 4. Origin of CYSLTR2 expression and characterisation of the immune microenvironment in uveal melanoma based on the single cell RNA expression analysis of the six tumours with $>2 \%$ CYSLTR2 $^{+}$ cells.

\section{Abbreviations}

FFPE: Formalin-Fixed Paraffin-Embedded; H\&E: Haematoxylin and Eosin; LUMC: Leiden University Medical Center; MAPK: Mitogen-Activated Protein Kinase; PCR: Polymerase Chain Reaction; PUM: Primary Uveal Melanoma; SNP: Single Nucleotide Polymorphism; TCGA: The Cancer Genome Atlas

\section{Acknowledgements}

We thank Nelleke A. Gruis (Department of Dermatology, LUMC) and Astrid G.S van Halteren (Department of Pediatrics, LUMC) for helpful discussions and reviewing the manuscript.

\section{Authors' contributions}

RJN and PAVV conceptualised this study and wrote the manuscript. MV, GPML, RMV, MCM and MJJ collected the patient materials. RJN, NVM and MV performed the PCR experiments, RJN performed the bioinformatic analyses, and RMV and MCM performed the histological examination. MV, RMV, MCM and MJJ revised the manuscript. PAVV supervised this study. All authors read and approved the final manuscript.

\section{Funding}

RJN is supported by the European Union's Horizon 2020 research and innovation program under grant agreement No 667787 (UM Cure 2020 project). MCM is supported by the Sydney Eye Hospital Foundation. The funders had no role in the design of this study, data collection and analysis, decision to publish, or preparation of this manuscript.

\section{Availability of data and materials}

The data sets analysed during the current study are available via the Broad GDAC Firehose (https://gdac.broadinstitute.org), NCl Genomic Data Commons data portal (GDC; https://portal.gdc.cancer.gov), Gene Expression Omnibus repository (https://www.ncbi.nlm.nih.gov/geo/query/acc.cgi?acc= GSE139829) and (supplementary) files of the original publications $[13,14,20]$.

\section{Ethics approval and consent to participate}

16 choroidal nevi were dissected from 13 formalin-fixed post-mortem human donor eyes, with consent from Lions NSW Eye Bank and approval from the University of Sydney and University of New South Wales Human Research Ethics Committee [7]. 120 fresh-frozen tumour samples, obtained from primary uveal melanomas treated by enucleation, were available from the Biobank of the Department of Ophthalmology, LUMC. This study was approved by the LUMC Biobank Committee and Medisch Ethische Toetsingscommissie under no. B14.003/DH/sh and B20.026. All patient samples were collected with informed consent, which was obtaind verbally as agreed by the medical ethical committee.

\section{Consent for publication \\ Not applicable.}

\section{Competing interests}

The authors declare that they have no competing interests.

\section{Author details}

${ }^{1}$ Department of Ophthalmology, Leiden University Medical Center, Leiden, the Netherlands. ${ }^{2}$ Department of Pathology, Leiden University Medical Center, Leiden, the Netherlands. ${ }^{3}$ Department of Pathology, Erasmus MC University Medical Center, Rotterdam, the Netherlands. ${ }^{4}$ Save Sight Institute and Department of Ophthalmology, University of Sydney, Sydney, Australia. ${ }^{5}$ School of Optometry and Vision Science, University of New South Wales, Sydney, Australia.

Received: 14 September 2020 Accepted: 1 February 2021

Published online: 15 February 2021

\section{References}

1. Virgili G, Gatta G, Ciccolallo L, Capocaccia R, Biggeri A, Crocetti E, et al. Incidence of uveal melanoma in Europe. Ophthalmology. 2007;114(12): 2309-15.

2. Singh AD, Borden EC. Metastatic Uveal Melanoma. Ophthalmol Clin. 2005; 18(1):143-50.

3. Coupland SE, Lake SL, Zeschnigk M, Damato BE. Molecular pathology of uveal melanoma. Eye (Lond). 2013;27(2):230-42.

4. Van Raamsdonk CD, Bezrookove V, Green G, Bauer J, Gaugler L, O'Brien JM, et al. Frequent somatic mutations of GNAQ in uveal melanoma and blue naevi. Nature. 2009;457(7229):599-602.

5. Van Raamsdonk CD, Griewank KG, Crosby MB, Garrido MC, Vemula S, Wiesner T, et al. Mutations in GNA11 in uveal melanoma. N Engl J Med. 2010;363(23):2191-9.

6. de Lange MJ, van Pelt SI, Versluis M, Jordanova ES, Kroes WG, Ruivenkamp $C$, et al. Heterogeneity revealed by integrated genomic analysis uncovers a molecular switch in malignant uveal melanoma. Oncotarget. 2015;6(35): 37824-35.

7. Vader MJC, Madigan MC, Versluis M, Suleiman HM, Gezgin G, Gruis NA, et al. GNAQ and GNA11 mutations and downstream YAP activation in choroidal nevi. Br J Cancer. 2017;117(6):884-7.

8. Johansson P, Aoude LG, Wadt K, Glasson WJ, Warrier SK, Hewitt AW, et al. Deep sequencing of uveal melanoma identifies a recurrent mutation in PLCB4. Oncotarget. 2016;7(4):4624-31.

9. Moore AR, Ceraudo E, Sher JJ, Guan Y, Shoushtari AN, Chang MT, et al. Recurrent activating mutations of G-protein-coupled receptor CYSLTR2 in uveal melanoma. Nat Genet. 2016:48(6):675-80.

10. Ceraudo E, Horioka M, Mattheisen JM, Hitchman TD, Moore AR, Kazmi MA, et al. 2019. https://doi.org/10.1101/663153.

11. Gusach A, Luginina A, Marin E, Brouillette RL, Besserer-Offroy E, Longpre JM, et al. Structural basis of ligand selectivity and disease mutations in cysteinyl leukotriene receptors. Nat Commun. 2019;10(1):5573.

12. Singh RK, Gupta S, Dastidar S, Ray A. Cysteinyl leukotrienes and their receptors: molecular and functional characteristics. Pharmacology. 2010; 85(6):336-49.

13. Robertson AG, Shih J, Yau C, Gibb EA, Oba J, Mungall KL, et al. Integrative analysis identifies four molecular and clinical subsets in Uveal melanoma. Cancer Cell. 2017;32(2):204-20 e15.

14. Durante MA, Rodriguez DA, Kurtenbach S, Kuznetsov JN, Sanchez MI, Decatur $\mathrm{CL}_{\text {, et }}$ al. Single-cell analysis reveals new evolutionary complexity in uveal melanoma. Nat Commun. 2020;11(1):496. 
15. van Eijk R, Stevens L, Morreau H, van Wezel T. Assessment of a fully automated high-throughput DNA extraction method from formalin-fixed, paraffin-embedded tissue for KRAS, and BRAF somatic mutation analysis. Exp Mol Pathol. 2013;94(1):121-5.

16. Versluis M, de Lange MJ, van Pelt SI, Ruivenkamp CA, Kroes WG, Cao J, et al. Digital PCR validates $8 \mathrm{q}$ dosage as prognostic tool in uveal melanoma. PLoS One. 2015;10(3):e0116371.

17. Zoutman WH, Nell RJ, van der Velden PA. Usage of droplet digital PCR (ddPCR) assays for T cell quantification in Cancer. Methods Mol Biol. 1884; 2019:1-14.

18. Garrison E, Marth G. Haplotype-based variant detection from short-read sequencing. arXiv preprint arXiv:12073907; 2012.

19. Li H, Handsaker B, Wysoker A, Fennell T, Ruan J, Homer N, et al. The sequence alignment/map format and SAMtools. Bioinformatics. 2009;25(16): 2078-9.

20. Anagnostou V, Bruhm DC, Niknafs N, White JR, Shao XM, Sidhom JW, et al. Integrative tumor and immune cell multi-omic analyses predict response to immune checkpoint blockade in melanoma. Cell Rep Med. 2020;1(8):100139.

21. Stuart T, Butler A, Hoffman P, Hafemeister C, Papalexi E, Mauck WM III, et al. Comprehensive Integration of Single-Cell Data. Cell. 2019;177(7): 1888-902.e21.

22. Wagle MC, Kirouac D, Klijn C, Liu B, Mahajan S, Junttila M, et al. A transcriptional MAPK pathway activity score (MPAS) is a clinically relevant biomarker in multiple cancer types. NPJ Precis Oncol. 2018;2(1):7.

23. Pollock PM, Harper UL, Hansen KS, Yudt LM, Stark M, Robbins CM, et al. High frequency of BRAF mutations in nevi. Nat Genet. 2002;33(1):19-20.

24. Yeh I, von Deimling A, Bastian BC. Clonal BRAF mutations in melanocytic nevi and initiating role of BRAF in melanocytic neoplasia. J Natl Cancer Inst. 2013;105(12):917-9.

25. Christodoulou E, Nell RJ, Verdijk RM, Gruis NA, van der Velden PA, van Doorn R. Loss of wild-type CDKN2A is an early event in the development of melanoma in FAMMM syndrome. J Invest Dermatol. 2020;140(11):2298-301 e3

26. Shain AH, Yeh I, Kovalyshyn I, Sriharan A, Talevich E, Gagnon A, et al. The genetic evolution of melanoma from precursor lesions. N Engl J Med. 2015; 373(20):1926-36.

27. Shain AH, Bagger MM, Yu R, Chang D, Liu S, Vemula S, et al. The genetic evolution of metastatic uveal melanoma. Nat Genet. 2019;51(7):1123-30.

28. Rodrigues M, Mobuchon L, Houy A, Alsafadi S, Baulande S, Mariani O, et al. Evolutionary routes in metastatic Uveal melanomas depend on MBD4 alterations. Clin Cancer Res. 2019;25(18):5513-24.

29. Karlsson J, Nilsson LM, Mitra S, Alsen S, Shelke GV, Sah VR, et al. Molecular profiling of driver events in metastatic uveal melanoma. Nat Commun. 2020;11(1):1894

30. Shain AH, Joseph NM, Yu R, Benhamida J, Liu S, Prow T, et al. Genomic and Transcriptomic Analysis Reveals Incremental Disruption of Key Signaling Pathways during Melanoma Evolution. Cancer Cell. 2018;34(1):45-55.e4.

31. Bollag G, Hirth P, Tsai J, Zhang J, Ibrahim PN, Cho H, et al. Clinical efficacy of a RAF inhibitor needs broad target blockade in BRAF-mutant melanoma. Nature. 2010;467(7315):596-9.

32. Slater K, Hoo PS, Buckley AM, Piulats JM, Villanueva A, Portela A, et al. Evaluation of oncogenic cysteinyl leukotriene receptor 2 as a therapeutic target for uveal melanoma. Cancer Metastasis Rev. 2018; 37(2-3):335-45.

33. Bronkhorst IH, Vu TH, Jordanova ES, Luyten GP, Burg SH, Jager MJ. Different subsets of tumor-infiltrating lymphocytes correlate with macrophage influx and monosomy 3 in uveal melanoma. Invest Ophthalmol Vis Sci. 2012;53(9): 5370-8

34. de Lange MJ, Nell RJ, Lalai RN, Versluis M, Jordanova ES, Luyten GPM, et al. Digital PCR-based T-cell quantification-assisted Deconvolution of the microenvironment reveals that activated macrophages drive tumor inflammation in Uveal melanoma. Mol Cancer Res. 2018;16(12): 1902-11.

35. Goto K, Pissaloux D, Paindavoine S, Tirode F, de la Fouchardiere A. CYSLTR2mutant cutaneous melanocytic neoplasms frequently simulate "pigmented Epithelioid Melanocytoma," expanding the morphologic Spectrum of blue tumors: a Clinicopathologic study of 7 cases. Am J Surg Pathol. 2019;43(10): 1368-76.

36. Slater K, Heeran AB, Garcia-Mulero S, Kalirai H, Sanz-Pamplona R, Rahman A, et al. High Cysteinyl Leukotriene Receptor 1 Expression Correlates with Poor Survival of Uveal Melanoma Patients and Cognate Antagonist Drugs
Modulate the Growth, Cancer Secretome, and Metabolism of Uveal Melanoma Cells. Cancers (Basel). 2020;12(10):2950.

37. Field MG, Durante MA, Anbunathan $H$, Cai LZ, Decatur CL, Bowcock AM, et al. Punctuated evolution of canonical genomic aberrations in uveal melanoma. Nat Commun. 2018;9(1):884-7.

38. Maat W, Jordanova ES, van Zelderen-Bhola SL, Barthen ER, Wessels HW, Schalij-Delfos NE, et al. The heterogeneous distribution of monosomy 3 in uveal melanomas: implications for prognostication based on fine-needle aspiration biopsies. Arch Pathol Lab Med. 2007;131(1):91-6.

\section{Publisher's Note}

Springer Nature remains neutral with regard to jurisdictional claims in published maps and institutional affiliations.
Ready to submit your research? Choose BMC and benefit from:

- fast, convenient online submission

- thorough peer review by experienced researchers in your field

- rapid publication on acceptance

- support for research data, including large and complex data types

- gold Open Access which fosters wider collaboration and increased citations

- maximum visibility for your research: over $100 \mathrm{M}$ website views per year

At $\mathrm{BMC}$, research is always in progress.

Learn more biomedcentral.com/submissions 